\title{
Endometrioid Stromal Sarcoma
}

National Cancer Institute

\section{Source}

National Cancer Institute. Endometrioid Stromal Sarcoma. NCI Thesaurus. Code C8973.

A malignant mesenchymal neoplasm that affects the uterine corpus, and rarely, the ovaries, cervix, and vagina. In the uterine corpus it is classified as low grade or high grade endometrial stromal sarcoma. In the remainder of the anatomic sites it is classified as low grade endometrioid stromal sarcoma. 